\title{
Olfactory Dysfunction is Associated with More Severe Clinical Course in COVID-19
}

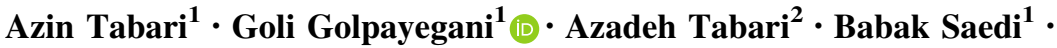 \\ Aydin Mahdkhah $^{1} \cdot$ Amin Amali $^{1} \cdot$ Saber Jazinizadeh $^{1} \cdot$ Leyla Sahebi $^{3}$. \\ Negin Saffarzadeh $^{4} \cdot$ Seyed Mousa Sadrehosseini ${ }^{1}$
}

Received: 21 October 2020/Accepted: 2 March 2021/Published online: 12 March 2021

(C) Association of Otolaryngologists of India 2021

\begin{abstract}
To perform a quantitative olfactory test in positive COVID19 RT-PCR admitted patients and asymptomatic ones, to evaluate the association between hyposmia and disease severity. This is a Cross sectional study. Ninety-one patients including 68 inpatients and 23 asymptomatic healthcare workers with positive COVID-19 RT-PCRs. Methods: Demographics and clinical characteristics were collected. Iran Smell Identification Test (IRSIT), a highly accurate 6-odorant test was used to evaluate
\end{abstract}

Goli Golpayegani

golpayegani.goli@gmail.com

Azin Tabari

atabari@sina.tums.ac.ir

Azadeh Tabari

atabari@mgh.harvard.edu

Babak Saedi

saedi@tums.ac.ir

Aydin Mahdkhah

aydinmahdkhah@gmail.com

Amin Amali

aminamali@yahoo.com

Saber Jazinizadeh

Saber.jazini@gmail.com

Leyla Sahebi

sahebileila@yahoo.com

Negin Saffarzadeh

neginsaffarzade68@gmail.com

Seyed Mousa Sadrehosseini

sadrehosseini@gmail.com

1 Department of Otolaryngology-Head and Neck Surgery, Otorhinolaryngology Research Center, Imam Khomeini Hospital Complex, Tehran University of Medical Sciences, Keshavarz Blvd, Tehran, Iran the reliability of self-reported hyposmia and determine the correlation of the measured olfactory dysfunction with disease severity. Twenty-two of 91 patients (24\%) reported hyposmia, while $41 / 91$ (45\%) patients had measurable olfactory dysfunction (IR-SIT score $1-4, p<0.05$ ). Mean age of the 68 inpatients and 23 asymptomatic patients were $43.97 \pm 16.13$ years; $\mathrm{M}: \mathrm{F} \quad 43: 25$, and $43.87 \pm$ 12.76 years; M:F 8:15 respectively. Of 68 patients, 20 were graded as severe, and 48/68 had mild course of disease. IR-

2 Department of Radiology, Massachusetts General Hospital and Harvard Medical School, Boston, MA, USA

3 Family Health Research Institute, Maternal-Fetal and Neonatal Research Center, Tehran University of Medical Science, Tehran, Iran

4 Department of Medical Genetics, School of Medicine, Tehran University of Medical Sciences, Tehran, Iran 
SIT detected hyposmia in $80 \%$ of patients with severe disease, and 50\% with mild disease, respectively. The risk of disease severity was significantly increased for patients with olfactory dysfunction and was detected 4 times higher when compared to patients with mild disease (OR 4, 95\% CI: $1.166-13.728, p=0.028$ ). Olfactory Dysfunction was present in $80 \%$ of patients with severe course. The risk of disease severity is significantly increased with olfactory dysfunction in admitted patients.

Keywords COVID-19 · SARS-CoV-2 · Hyposmia · Olfactory dysfunction · Olfactory test

\section{Introduction}

There is rapidly accumulating anecdotal evidence that anosmia (loss of smell) is frequently reported symptom associated with the COVID-19 pandemic. Previously described human strains of coronavirus have also been demonstrated to invade the central nervous system and propagate from within the olfactory bulb [1-3].It is therefore perhaps no surprise that alterations in sense of smell would be reported by patients with COVID-19. Multiple reports are surfacing from countries around the world including Italy, Iran and Spain showing the sudden surge in the number of patients presenting with anosmia [4-10]. COVID-19 is now recognized to be highly heterogeneous in severity-ranging from asymptomatic (or symptoms too mild to be noticed) to severe acute respiratory distress syndrome and death-and in symptomatology, which extends to include otolaryngologic symptoms. Patients with the well-known and characteristic symptoms of COVID-19 may be readily identified (by themselves or others). There have been reports that olfactory dysfunction can be the primary or the only symptom, therefore, patients experiencing nonclassical symptoms of COVID-19 may be missed [9-12].In this study we have investigated the reliability of subjective reports of smell loss and the correlation of olfactory dysfunction with severity of COVID-19.

\section{Methods}

The study protocol was approved by the local ethics committee and the National Ministry of Health (license number IR.TUMS.VCR.REC.1399.305). Detailed information about the study was given to the participants, and an informed written consent was obtained from each participant.

\section{Subjects}

This study included 91 individuals from June 2020 to July 2020. All the subjects were divided into 2 groups: 1-patients who had confirmed COVID-19 with RT-PCR of nasopharyngeal and throat, done based on the World Health Organization (WHO) recommendation [13], and were admitted to a tertiary COVID referral hospital $(n=68), 2$ - asymptomatic health care workers who, were screened as part of hospital protocol, and were positive for COVID-19 RT-PCR $(n=23)$. The inclusion criteria comprised: hemodynamic stability, age $>18$ years, understand Farsi and ability to follow the testing instructions. Patients with history of head trauma, skull base fracture, neoplasms of nose, sinus or brain, exposure to irritant chemical cleansers, prior olfactory dysfunction, prior neurological disorders that can affect olfactory function, nasal polyposis and acute sinusitis were excluded.

\section{Clinical Severity Classification of COVID-19 Patients}

COVID-19 detection protocol of National Ministry of Health was used which includes CT imaging (64 slice high speed CT, GH Healthcare) with positive findings. Moreover, the diagnosis of COVID-19 disease was confirmed by quantitative detection of SARS-CoV-2 RNA using RTPCR (viral nucleic acid extraction kit, RBCBioscience Inc., Brussels, Belgium) of the patients' nasopharyngeal wash/ aspirate or nasal aspirate. The severity of COVID-19 was categorized as mild $(\mathrm{SpO} 2>90 \%$ with or without risk factors), and severe (patients with $\mathrm{SPO} 2<90 \%$ at initial presentation or progressive disease course during hospital stay; drop in SPO2, ICU admission, intubation, acute respiratory distress syndrome). Age $>55$ years old, pre-existing pulmonary disease, chronic kidney disease, diabetes with A1c $>7.6 \%$, history of hypertension or cardiovascular disease or transplant or immunosuppression or HIV were considered as epidemiological risk factors. Critical vital signs were respiratory rate $>24$ breaths $/ \mathrm{min}$, heart rate $>125$ beats/min and $\mathrm{SpO} 2<90 \%$ on ambient air. In lab findings, D-dimer $>1000 \mathrm{ng} / \mathrm{mL}, \mathrm{CPK}>$ twice upper limit of normal, CRP $>100, \mathrm{LDH}>245 \mathrm{U} / \mathrm{L}$, elevated troponin, admission absolute lymphocyte count $<0.8$ and ferritin $>300 \mathrm{ug} / \mathrm{L}$.

\section{Subjective Evaluation of Loss of Sense of Smell}

All subjects were asked to fill out a questionnaire including medical conditions, medications, prior olfactory problems, smoking habit, suspicious recent close contacts, current symptoms, duration of symptoms and contact information. 


\section{Objective Evaluation of Olfactory Function}

The olfactory function was assessed using the quick IR-SIT [14]. IR-SIT is the Iranian verified version of UPSIT, using six odors including banana, rosewater, cinnamon, garlic, mint, and melon, with high accuracy in differentiating anosmia, hyposmia and normosmia. Six odors were presented to the patient as a sticker. Stickers were scratched by the physician and patients were asked to smell them from 2-cm distance. Patients had to select the correct odor name from the 4-alternative test sheet. Patients with a total score of 5 and 6 (number of odorants diagnosed correctly) were considered normosmic, while hyposmic patients were scored 1-4. Anosmia was defined as 0 .

\section{Assessment of Olfactory Dysfunction and Severity of the Disease}

All patients were divided into two groups with normal sense of smell and hyposmia using IR-SIT testing. We then compared the 2 groups in terms of demographic, comorbidity variables and severity of the disease.

\section{Statistical Analysis}

Chi-square test was used for comparison of categorical variables. Independent sample t-test was used for comparison of means. The association of COVID-19 severity and hyposmia was determined using logistic regression. The agreement between subjective hyposmia and objective test was performed by Phi test. $P$ values less than 0.05 were considered statistically significant. Statistical analyses were performed using SPSS 24.

\section{Results}

This cohort included 91 individuals (51 males, 40 females; mean age, $43.95 \pm 15.28$ years old; age range, 15-82 years old) from June 2020 to July 2020. Subjects' characteristics and demographics are summarized in Table 1. The COVID-19 symptomatic patients' most common presenting symptoms were cough $(n=45)$, dyspnea $(n=40)$, fever $(n=37)$, myalgia $(n=33)$ and sore throat $(n=17)$. As shown in Table 1 , inpatients tend to have more comorbidities when compared to asymptomatic individuals $(p<0.001)$. Inpatients were significantly more hypertensive comparing to asymptomatic individuals ( $p=0.03$ ). In addition, COVID-19 positive patients with negative smoking history, no diabetes mellites and no hypertension were more likely to remain asymptomatic when compared to the inpatient group (Table 1).
$10 / 23(43.5 \%)$ of asymptomatic individuals being positive screened for COVID-19 has reported hyposmia in history taking but their objective test results were normal. Interestingly, only $1 / 23(4 \%)$ of asymptomatic subjects were considered hyposmic when performed the objective olfactory test.

All 68 inpatients were divided into two groups with normal sense of smell $(n=28)$ and hyposmia $(n=40)$ using objective olfactory testing (Table 2). Fifty-eight percent $(n=40)$ of inpatients with COVID-19 had measured olfactory dysfunction and were scored 1-4 (hyposmia) on IR-SIT test; 10(25.0\%) had severe smell dysfunction (IR-SIT score $=1-2$ ) and 30(75.0\%) patients had mild to moderate olfactory dysfunction (IR-SIT score $=3-4$ ), while there were no pure anosmic subjects. The IR-SIT testing revealed that the admitted patients exhibited marked olfactory dysfunction compared to asymptomatic individuals (40/68 and $1 / 23$ respectively, $p=0.001$ ).

Generally, 34 cases of inpatients (60.7\%) who did not report hyposmia, were classified as some degrees of olfactory dysfunction recognized by IR-SIT test. Inpatients with hyposmia under-reported their loss of sense of smell by $70 \%$ (12/68 self-report vs. $40 / 68$ with objective olfactory testing). Therefore, there is no significant agreement between subjective hyposmia and objective test results among inpatients $(\mathrm{Phi}$ Test value $=-0.083, \quad p$ value $=0.434)$.

Of 68 inpatients, 20 and 48 patients had severe and mild disease, based on the criteria such as $\mathrm{SPO}_{2}$, vital signs, risk factors and their respiratory progressive disease course during their hospital stay. It is important to point out that $80 \%(16 / 20)$ of patients with more progressive disease had objective olfactory dysfunction whereas, only 50\% (24/48) of mild patients had objective hyposmia. The risk of disease severity was significantly increased in patients with olfactory dysfunction and was detected at 4 times higher rate, when compared to patients with mild disease (OR 4, 95\% CI: $1.166-13.728, p=0.028$ ).

\section{Discussion}

This study quantitatively evaluated olfaction in a sizable cohort of patients diagnosed with the SARS-CoV-2 virus infection. By employing a well-validated 6-item smell test [14], COVID-19 patients were able to be classified into distinct categories of olfactory dysfunction, with 40 of 68 (60\%) admitted patients and 1 of $23(1.7 \%)$ asymptomatic healthcare workers with confirmed COVID-19, exhibiting hyposmia. In the present study, only (13/91) $14 \%$ of the subjects were aware of their olfactory deficit before testing, which is a lower percentage compared to previous reports 
Table 1 Characteristics and demographics of the 2 groups

\begin{tabular}{llll}
\hline & Inpatients (68) & Asymptomatic individuals (23) & $P$ value \\
\hline Mean Age (years) \pm SD & $43.97 \pm 16.13$ & $43.87 \pm 12.76$ & \\
Sex; n (\%) & & & \\
Female (40) & $25(62.5 \%)$ & $15(37.5 \%)$ & 878 \\
Male (51) & $43(84.3 \%)$ & $8(15.6 \%)$ & \\
Smoking history; n (\%) & & $1(11.2 \%)$ & 0.011 \\
Yes & $8(88.8 \%)$ & $22(26.8 \%)$ & $10(45.4 \%)$ \\
No & $60(73.1 \%)$ & $1(2.4 \%)$ & $3(6.9 \%)$ \\
Smell complaints & $12(54.5 \%)$ & $1(7.2 \%)$ \\
Smell dysfunction(IR-SIT) & $40(97.6 \%)$ & 0 & $<0.001$ \\
Comorbidities & $41(93.1 \%)$ & 0 & $0.108^{*}$ \\
Diabetes & $13(92.8 \%)$ & 001 \\
Hypertension & $12(100 \%)$ & $1(25 \%)$ \\
Cancer & $2(100 \%)$ & $1 *$ \\
Chronic kidney disease & $1(100 \%)$ & $1 *$ \\
Chronic pulmonary disease & $3(75 \%)$ & $1 *$ \\
\hline
\end{tabular}

*P value based on fisher exact test

with interviews from inpatients or online surveys (34\% and $60)[15,16]$. Since the first published studies about olfactory dysfunction surge during COVID-19 pandemic, different studies have investigated it's prevalence through population based online surveys $(60 \%)$ [16], self-report or questionnaire in outpatient (47\%) [17] or inpatients (5.1\%, $33.9 \%, 85.6 \%)[15,18,19]$ and validated smell test $(98 \%)$ [20]. One of the main reasons resulting in the gap between self-report rate and quantified smell assessment is that in general, anosmia is more noticeable compared to hyposmia. Therefore, many confirmed patients may not report hyposmia. The difference between self-report rate and quantified smell assessment proved the importance of performing a quantitative olfactory dysfunction test for all the patients regardless of their complaint.

Ninety one percent of admitted patients did not present with nasal obstruction or rhinorrhea. This leads to suspecting another reason for hyposmia than mechanical nasal obstruction. SARS-COV-2 may enter the CNS through retrograde neuronal route that is supported by the fact that some patients develop hyposmia [21, 22]. This phenomenon may also be indicative of the immunosuppression in COVID-19 patients with CNS symptoms, especially in the severe subgroup [22]. Hyposmia showed significant correlation with severity of the disease in the inpatient group. We observed that $80 \%$ of patients with more severe course of the disease presented with olfactory dysfunction. This is consistent with a previous study that pointed a correlation between neurologic symptoms such as anosmia and COVID-19 disease severity in inpatient setting
[21, 23-27]. Occurrence of olfactory dysfunction during other viral infections has strong correlation with disease severity [18, 19, 25-28]. Hyposmia as a neurologic symptom may need for further close follow-up in inpatients. In addition, neuro-invasion may increase the risk of multiple sclerosis and Parkinson in future [22].

The strengths of this study were using quantitative test to evaluate olfactory function which is more sensitive instead of relying on self-reports or subjective questionnaires. Moreover, we included both symptomatic and asymptomatic patients which makes it a unique study population. To our knowledge, this is the first study designed to evaluate if loss of smell has a prognostic significance on the disease severity.

This study has some limitations. First, the low number of subjects due to the unknown nature of COVID-19 in the first months of pandemic. The low number of cases were directly the result of safety issue concerns in critical care. We included the data of the admitted patients, therefore the symptomatic ambulatory patients who showed milder disease were excluded since they mostly preferred to stay at home during quarantine. Second, examiners were not blinded to the diagnosis when performing the olfactory test. Lastly, we were unable to perform the objective test on the more severely ill such as ICU patients, due to safety precautions.

Based on our findings, objective smell testing enables confirmatory diagnosis in the absence of other symptoms and helps to predict the clinical course of COVID-19 in terms of progression and severity. We would also like to 
Table 2 Baseline characteristics and Objective test results of the admitted patient group

\begin{tabular}{|c|c|c|c|}
\hline & Normal sense of smell (28) & Hyposmia (40) & $P$-value \\
\hline Age; Mean (SD) & $43.32 \pm 14.74$ & $44.43 \pm 17.20$ & 0.784 \\
\hline $\operatorname{Sex}(F: M)$ & $13: 15$ & $12: 28$ & 0.167 \\
\hline Positive smoking history (\%) & $2(25 \%)$ & $6(75 \%)$ & $0.455^{* *}$ \\
\hline Comorbidities & $15(36.5 \%)$ & $26(63.4 \%)$ & 0.343 \\
\hline Diabetes & $4(30.8 \%)$ & $9(69.2 \%)$ & 0.397 \\
\hline Hypertension & $2(16.7 \%)$ & $10(83.3 \%)$ & $0.084 * *$ \\
\hline \multicolumn{4}{|l|}{ Symptoms } \\
\hline Cough & $18(40 \%)$ & $27(60 \%)$ & 0.873 \\
\hline Dyspnea & $17(42.5 \%)$ & $23(57.5 \%)$ & 0.791 \\
\hline Fever & $18(48.6 \%)$ & $19(51.4 \%)$ & 0.171 \\
\hline Myalgia & $16(48.5 \%)$ & $17(51.5 \%)$ & 0.187 \\
\hline Sore throat & $7(41.2 \%)$ & $10(58.8 \%)$ & 1 \\
\hline Dysgeusia & $8(61.5 \%)$ & $5(38.5 \%)$ & 0.097 \\
\hline Hyposmia & $6(50 \%)$ & $6(50 \%)$ & 0.494 \\
\hline Sneezing & $1(20 \%)$ & $4(80 \%)$ & $0.642 * *$ \\
\hline Rhinorrhea & $1(25 \%)$ & $3(75 \%)$ & 0.498 \\
\hline Nasal obstruction & 0 & $6(100 \%)$ & 0.032 \\
\hline Diarrhea & $10(47.6 \%)$ & $11(52.4 \%)$ & 0.471 \\
\hline Nausea/vomiting & $11(55 \%)$ & $9(45 \%)$ & 0.135 \\
\hline \multicolumn{4}{|l|}{ Signs } \\
\hline $\mathrm{SpO} 2$ & & & 0.077 \\
\hline$<90 \%$ & $8(28.6 \%)$ & $20(71.4 \%)$ & \\
\hline $90 \%$ & $20(50 \%)$ & $20(50 \%)$ & \\
\hline CRP & & & 0.961 \\
\hline 100 & $7(41.2 \%)$ & $10(58.8 \%)$ & \\
\hline$<100$ & $18(41.9 \%)$ & $25(58.1 \%)$ & \\
\hline Lymph count & & & 0.155 \\
\hline$<0.8$ & $9(31 \%)$ & $20(69 \%)$ & \\
\hline $0.8<$ & $17(48.6 \%)$ & $18(51.4 \%)$ & \\
\hline
\end{tabular}

*p-value based on t-test

*** p-value based on fisher exact test

$\mathrm{SpO} 2$ = Oxygen saturation; $\mathrm{CRP}=\mathrm{C}$-reactive protein; Lymph count $=$ absolute lymphocyte count

draw attention to the importance of objective assessment of olfactory dysfunction. It is important to perform the testing in early stages, regardless of patient complaint of loss of sense of smell. It is time for public health workers to recognize the link between COVID-19 severity and smell disturbances.

\section{Key messages}

COVID-19-related olfactory dysfunction is a known description in the medical literature. Overall, $60 \%$ of the patients with COVID-19 present with hyposmia. Olfactory dysfunction is significantly associated with more progressive course of the disease in current study. The outcome is consistent in $80 \%$ of patients with severe disease. This notion may need to be communicated to the medical community.

Funding This research received no specific grant from funding agency in the public, commercial, or non-for-profit sectors.

\section{References}

1. Dubé M, Le Coupanec A, Wong AH, Rini JM, Desforges M, Talbot PJ (2018) Axonal transport enables neuron-to-neuron propagation of human coronavirus OC43. J Virol 92 (17) 
2. Desforges M, Le Coupanec A, Stodola JK, Meessen-Pinard M, Talbot PJ (2014) Human coronaviruses: viral and cellular factors involved in neuroinvasiveness and neuropathogenesis. Virus Res 194:145-158

3. Morris M, Zohrabian V (2020) Neuroradiologists, be mindful of the neuroinvasive potential of COVID-19. Am J Neuroradiol 41:E37-E39. https://doi.org/10.3174/ajnr.A6551

4. Cheng Q, Yang Y, Gao J (2020) Infectivity of human coronavirus in the brain. Biomedicine 56:102799

5. Hopkins C, Surda P, Kumar N (2020) Presentation of new onset anosmia during the COVID-19 pandemic. Rhinology 58(3):295-298

6. Acter T, Uddin N, Das J, Akhter A, Choudhury TR, Kim S (2020) Evolution of severe acute respiratory syndrome coronavirus 2 (SARS-CoV-2) as coronavirus disease 2019 (COVID-19) pandemic: a global health emergency. Sci Total Environ 730:138996

7. Heidari F, Karimi E, Firouzifar M, Khamushian P, Ansari R, Ardehali MM, Heidari F (2020) Anosmia as a prominent symptom of COVID-19 infection. Rhinology 58(3):302-303

8. Krajewska J, Krajewski W, Zub K, Zatoński T (2020) COVID-19 in otolaryngologist practice: a review of current knowledge. Eur Arch Oto-Rhino-Laryngol 277(7):1885-1897

9. Gane SB, Kelly C, Hopkins C (2020) Isolated sudden onset anosmia in COVID-19 infection. A novel syndrome. Rhinology 58(3):299-301

10. Cao Y-C, Deng Q-X, Dai S-X (2020) Remdesivir for severe acute respiratory syndrome coronavirus 2 causing COVID-19: An evaluation of the evidence. Travel Med Infect Dis 35:101647

11. Nicola M, O'Neill N, Sohrabi C, Khan M, Agha M, Agha R (2020) Evidence based management guideline for the COVID-19 pandemic-review article. Int J Surg 77:206-216. https://doi.org/10.1016/j.ijsu.2020.04.001

12. Gilani S, Roditi R, Naraghi M (2020) COVID-19 and anosmia in Tehran Iran. Med Hypotheses 141:109757

13. WHO (2020) Laboratory testing for coronavirus disease (COVID-19) in suspected human cases: interim guidance, 19 March 2020. World Health Organization

14. Kamrava SK, Jalessi M, Ghalehbaghi S, Amini E, Alizadeh R, Rafiei F, Moosa S, Farhadi M (2020) Validity and reliability of persian smell identification test. Iranian J Otorhinolaryngol 32(109):65

15. Giacomelli A, Pezzati L, Conti F, Bernacchia D, Siano M, Oreni L, Rusconi S, Gervasoni C, Ridolfo AL, Rizzardini G (2020) Self-reported olfactory and taste disorders in patients with severe acute respiratory coronavirus 2 infection: a cross-sectional study. Clin Infect Dis 71:889-890. https://doi.org/10.1093/cid/ciaa330

16. Bagheri SH, Asghari A, Farhadi M, Shamshiri AR, Kabir A, Kamrava SK, Jalessi M, Mohebbi A, Alizadeh R, Honarmand AA (2020) Coincidence of COVID-19 epidemic and olfactory dysfunction outbreak in Iran. Med J Islamic Repub Iran 34:62

17. Spinato G, Fabbris C, Polesel J, Cazzador D, Borsetto D, Hopkins C, Boscolo-Rizzo P (2020) Alterations in smell or taste in mildly symptomatic outpatients with SARS-CoV-2 infection. JAMA 323:2089. https://doi.org/10.1001/jama.2020.6771

18. Besser G, Liu DT, Renner B, Hummel T, Mueller CA (2020) Reversible obstruction of the olfactory cleft: impact on olfactory perception and nasal patency. In: International forum of allergy \& rhinology. Wiley Online Library 6:713-718

19. Lechien JR, Chiesa-Estomba CM, De Siati DR, Horoi M, Le Bon SD, Rodriguez A, Dequanter D, Blecic S, El Afia F, Distinguin L (2020) Olfactory and gustatory dysfunctions as a clinical presentation of mild-to-moderate forms of the coronavirus disease (COVID-19): a multicenter European study. Eur Arc Oto-RhinoLaryngol 277:1-11

20. Moein ST, Hashemian SM, Mansourafshar B, Khorram-Tousi A, Tabarsi P, Doty RL (2020) Smell dysfunction: a biomarker for COVID-19. In: International forum of allergy \& rhinology. Wiley Online Library 8:944-950

21. Mao L, Jin H, Wang M, Hu Y, Chen S, He Q, Chang J, Hong C, Zhou Y, Wang D (2020) Neurologic manifestations of hospitalized patients with coronavirus disease 2019 in Wuhan, China. JAMA Neurol 77(6):683-690

22. Das G, Mukherjee N, Ghosh S (2020) Neurological insights of COVID-19 pandemic. ACS Chem Neurosci 11(9):1206-1209

23. Montalvan V, Lee J, Bueso T, De Toledo J, Rivas K (2020) Neurological manifestations of COVID-19 and other coronavirus infections: a systematic review. Clinical Neurol Neurosurg 194:105921

24. Román GC, Spencer PS, Reis J, Buguet A, Faris MEA, Katrak SM, Láinez M, Medina MT, Meshram C, Mizusawa H (2020) The neurology of COVID-19 revisited: a proposal from the Environmental Neurology Specialty Group of the World Federation of Neurology to implement international neurological registries. J Neurol Sci 414:116884

25. Yan CH, Faraji F, Prajapati DP, Ostrander BT, DeConde AS (2020) Self-reported olfactory loss associates with outpatient clinical course in COVID-19. In: International forum of allergy \& rhinology. Wiley Online Library 7:821-831

26. Kotfis K, Williams Roberson S, Wilson JE, Dabrowski W, Pun BT, Ely EW (2020) COVID-19: ICU delirium management during SARS-CoV-2 pandemic. Crit Care 24:1-9

27. Munhoz RP, Pedroso JL, Nascimento FA, Almeida SMD, Barsottini OGP, Cardoso FEC, Teive HAG (2020) Neurological complications in patients with SARS-CoV-2 infection: a systematic review. Arq Neuropsiquiatr 78(5):290-300

28. Trotier D, Bensimon JL, Herman P, Tran Ba Huy P, Døving KB, Eloit C (2007) Inflammatory obstruction of the olfactory clefts and olfactory loss in humans: a new syndrome? Chem Senses 32(3):285-292

Publisher's Note Springer Nature remains neutral with regard to jurisdictional claims in published maps and institutional affiliations. 